its actual size. It is, more properly speaking, a tuboovarian retention cyst, inasmuch as the ovary forms part of the cyst wall, and the contents consist of the retained menstrual fluid. Fig. 2 represents the right distended tube and ovary, two-thirds its actual size. The ovary is distinct by itself and in a condition of cystic degeneration. The Fallopian tube and adventitious cysts at its end contain menstrual fluid. The plates are accurate, the drawings being made before the cysts were opened, so I shall not describe the external appearance of the two specimens.

The pathologic anatomy and nature of the retained menses are well known. In this connection let me say that two fimbriæ of the left tube could be differentiated within the cyst, and the contents were free from germs.

\section{THE USE OF THE CURETTE IN ACUTE INFECTION OF UTERUS WITH ADHERENT PLACENTA.}

Presented to the Section on Obstetrics and Diseases of Women at the Forty-ninth Annual Meeting of the American Medical Assoeiation, held at Denver, Colo., July $\mathbf{7 - 1 0 , 1 8 9 8 . ~}$

BY D. S. FAIRCHILD, M.D.

$$
\text { cLrNTON, IOWA. }
$$

In the midst of the discussions of the more difficult and complex questions of abdominal and pelvic surgery our leading and most eminent gynecologists, whose opinions are of inestimable value to workers in more limited fields of general gynecologic work, often fail to furnish us lessons from the results of their own varied experience in the less brilliant but equally important matters pertaining to every-day practice.

I have often been impressed with the thought, if our information was definite enough in relation to the use of the curette in removing attached portions of placenta in cases of abortion, premature labor or labor at full term when infection has occured. It is clear enough that in diseases of the endometrium the curette is of great value and is, as a general rule, properly used in the cases which come under our daily notice. But in the cases where, from the retention of small masses of adherent placenta, infection has occurred, the uterine mucosa is softened, the uterine lymphatics are blocked with infectious thrombi and the temperature elevated from absorption of septic material, is it really safe to employ the curette in a vigorous and indiscriminate manner? I have frequently observed in general surgical practice that in certain cases of local infection anything more than the most careful and delicate procedures has been attended by a sudden accession of the local symptoms, together with a marked rise of temperature and have observed the same condition arise after curetting a uterus containing acute infective material.

I am aware that many advocate the use of the curette in septic conditions of the uterus following abortions or after labor, when there is reason to believe that portions of placenta are still retained in the organ. I have of late years been led to believe that this is not an altogether safe practice to follow. Great discrimination should be employed in resorting to this procedure, otherwise much harm may be done.

In cases where decomposing material affected by saprophytic micro-organisms is retained in the uterus the influence of which is marked by high temperature and offensive lochia, the dull curette employed carefully for the purpose of removing from the organ the offending substance, which has but little or no attach. ments to the uterine walls, is an exceedingly valuable procedure, followed by irrigation with sterilized water. When, however, a septic endometritis exists in connection with necrotic decidua burdened with septic germs, streptocccci, the curette may become a very dangerous instrument. In these cases it is quite easy to inflict a trauma which may serve as an additional means of introducing septic germs into the system. The protective granulation layer which serves a conservative purpose of limiting the constitutional infec. tion may thus be distributed, furthermore it is quite possible to penetrate the softened uterine tissue and thus lead to most disastrous results.

The use of the curette in the removal of adherent portions of placenta in the early period after an abortion, before a septic inflammation of the uterus has occurred is beneficial and this together with thorough irrigation will save some of the danger of infection. But after septic infection has once developed this treatment is attended, with considerable danger as I have abundant proof in my own hands and in the practice of professional friends. I have no recent misfortune of my own to record for I have long since abandoned this method of treatment. The removal of adherent masses under these conditions by scraping with the finger nail is not less hazardous, but oven more so, on account of the trauma inflicted and on account of the risk of introducing germs which may lie concealed under the nail, especially under some nails which may be used for this purpose. The conditions which we have to contend with in these cases are purely surgical and should be treated accordingly. If a surgeon, in the treatment of an infective wound, should undertake to remove necrotic tissue by violent means be would find that the condition of the wound and the general condition of the patient would become more serious. In all cases of septic infection, whether of wounds or of the uterus, drainage is the first consideration together with the removal of infective material so far as possible without disturbing the boundaries which conservative efforts have placed for the purpose of protecting the system against infection. It often becomes a delicate question what to do in cases of sepsis arising from the uterus. The general practitioner will, in a great number of cases, be called upon to decide what course to pursue, and if he has in mind the necessity of employing the curette as the most approved method of treatment he will sometimes be led into most serious difficulty and do his patient great harm. A correct appreciation of the pathologic condition should underlie the measures to be employed. If the examination reveals the existence of necrotic decidua, bearing saprophytic microorganisms, giving rise to sapremic intoxication, the careful use, of the dull curette, not for the purpose of scraping the interior of the uterus, but for the purpose of facilitating the escape of the contents of the organ, followed by irrigation with sterilized water, will accomplish the desired end. If, as it is sometimes found, the os is somewhat tightly closed, under the influence of an anesthetic the cervix can easily be placed in the most favorable position and sufficiently dilated for satisfactory drainage. If it is found however that the uterus is swollen and the endometrium softened and infected with streptococcus, only the irrigation should be employed, and the irrigator handled with great care lest the violence disturb the granulation layer and expose new points for the ab- 
sorption of infection and thus expose the system to a larger dose of poison. If the examination reveals the absence of an offensive discharge, and the general symptoms are profound and not attended with local conditions other than a septic and necrotic endometrium, no local treatment should be instituted, for it is probable that the uterus is infected throughout, that a condition of thrombo phlebitis exists, that the uterus is softened, that no granulation layer exists, and the use of the curette or irrigation can do no good, but rather harm, and should be avoided. Whatever may be the merits of a vaginal hysterectomy in these cases can not be considered in this paper for the reason that the great multitude of physicians who have these patients to treat can not afford to employ it in general. It will occasionally happen that the os is closed in cases in which it will be necessary to reach the interior of the organ. I do not remember of meeting with a case where I could not dilate the cervix with my finger when the patient was under the influence of an anesthetic, but if the cervix is too rigid to be dilated in this manner forcible instrumental means may be employed, but great care should be observed lest a trauma be inflicted and at least afford additional facilities for the absorbtion of septic material.

This paper is presented simply as a protest against what I believe to be a indiscriminate use of the curette in acute infection of the uterus. While these observations may not have any application to leading members of the profession I am quite sure that the lines bounding the use of this instrument are not often enough drawn to furnish $\operatorname{saf} \theta$ guides for many practitioners whose experience is more limited.

I contend that great care should be observed in the use of the curette in acute infection of the uterus.

In cases in which portions of placenta are adherent they should be removed by the sharp curette, but before infection has occurred, under antiseptic precautions. If infection has developed, it is safer to remove all detached portions with a dull curette without inflicting trauma, irrigate and drain. If infection of the uterus has occurred without offensive lochia but with serious constitutional symptoms, no curettement or irrigation should be employed. The dangers of the free use of the curette are of two kinds: 1, inflicting trauma which may facilitate absorption of infection material; 2, the penetration of the softened uterine tissue. If the os has closed, retaining in the uterus suspicious or decomposing material, very careful dilatation should be employed under an anesthetic; detached masses removed with the dull curette and gentle irrigation employed; the whole procedure so conducted as to inflict the least trauma possible.

\section{THE EFFECT OF UTERINE CURETTAGE AND DRAINAGE ON DISEASE OF THE TUBES.}

Presented to the Section on Obstetrics and Discases of Women at the Forty-ninth Annual Meeting of the American Medical Association, held at Denver, Colo., June 7-10, 1897.

BY W. W. GRANT, M.D.

DENVER, COLO.

One of the commonest diseases of womanhood, and at the same time one of the most serious in its consequences to health and life, is inflammation of the Fallopian tubes. It is very generally, if not uniFallopian tubes. It is very generally, if not uni-
formly, secondary to disease elsewhere. Like peri-
orrheal, as well as puerperal or other infection. It tonitis, it is not idiopathic. It is a common cause of sterility, either in direct consequence of the disease, or as a result of the surgical measures employed for its cure. It leads not infrequently to fatal results. Therefore to prevent mortality and to preserve mental stability and the functions of maternity, disease of these appendages is second in importance to no other, and appeals always to our highest consideration and best judgment.

It is just to say that pyosalpinx is a purely surgical condition, requiring some form of operative procedure for its permanent cure, yet cases now and then recover without recourse to the knife. As uterine endometritis is the usual cause of salpingitis, it is self-evident that whatever means will prevent, or most speedily cure, the former disease can only tend to the preservation of the functions and integrity of the tubes. Acute aseptic corporeal endometritis is not often observed. As sepsis following abortion, labor, gonorrhea or operations, is the usual cause of serious forms of uterine endometritis, and secondarily of salpingitis, it follows that prompt and efficient treatment of the endometritis is a primary necessity of the greatest importance. In gonorrheal endometritis the vir. ulence of the gonococci is not, at this stage, so great, being rarely acute even at a comparatively early period, and is not therefore manifested by the severe positive symptoms of the acute vulvo-vaginal, uretheral and cervical forms of the disease, consequently it is generally overlooked. To Sanger is credited the statement that about one-eighth of all gynecologic diseases are credited to gonorrhea. Unlike puerperal sepsis there are no immediate constitutional or peritoneal indications, these being dependent upon septic absorption as well as extension of the disease in one case, and chiefly extension of the inflammation to the tubes in the gonorrheal form, and usually by leakage from the abdominal ostium of the tube inducing severe violent or local peritonitis. In every form of septic endometritis except the early gonorrheal, the wise and accepted rule of treatment is to clean out all débris and foci of infection from the uterus by curettage, irrigation, or both, ordinarily supplemented by gauze drainage. The rule is the reverse, in fact the treatment is negative, as to the early period of gonorrheal endometritis, on the ground that to curette the uterus at this time and in this condition is to open up new channels of infection and cause a more certain extension of disease to the tubes and peritoneum. I very much doubt the wisdom of the usual inaction and delay at so important a juncture. The reasoning I believe more theoretic than practical. The uterus is not in the soft receptive state of the puerperium, and yet we know that the mortality in the latter has been greatly diminished and should practically be abolished by early curettage, irrigation and gauze drainage. Deep and thorough curettage is specially necessary in the gonorrheal cases, because the utricular glands are deeply affected. After curretting apply immediately such a mixture as iodin and carbolic acid, and then antiseptic gauze for drainage and there need be liitle fear of aggravating the disease. The surgical principle involved is the same in all-to remove the diseased tissue, which is the source of infection, and place the parts in the best possible contion for drainage and thus prevent further infection. This is the dictum of modern surgery and I believe should be applied in the early period of uterine gon- 